\title{
BIOMECHANICS OF PENETRATING TRAUMA AND INJURY MITIGATING PROTECTIVE MEASURES IN GUARDRAIL MOTOR VEHICLE CRASHES
}

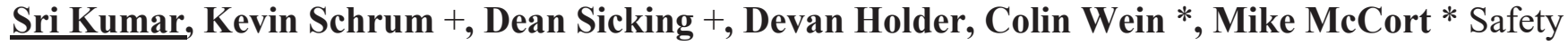 \\ Research Institute, Atlanta, GA \\ + University of Alabama, Birmingham, AL \\ * Compass Consulting Engineers, Westminster, CO
}

Corresponding Author: Dr. Sri Kumar

Email:kumar@srinst.com

https://doi.org/10.34107/BiomedSciInstrum.57.04128

\begin{abstract}
The objective of the present study is two-fold. First, the elucidation of the biomechanics of penetrating trauma as a result of guardrail intruding into the occupant compartment. Second, the evaluation of the biomechanical efficacy of hybrid tensioncompression guardrails to better protect occupants. The nine fatally guardrail penetrating crashes occurred between 2016 and 2019 were analyzed to study the mechanism of injuries. Four car-to-guardrail crash tests were conducted using a hybrid guardrail that integrated the commonly used W-beam with a new design of tension-based end terminal. The test included the impact of a bogey-type platform, small sedan vehicles, and a pick-up truck at highway speeds onto the guardrail. The impact orientation was varied to simulate the frontal and oblique corner crashes with a speed ranging from 90 to $111 \mathrm{kph}$. The realworld studies showed that the fatal injuries were due to impaling guardrail regardless of vehicular speed and size. The occupants not in the trajectory of the guardrail in the same vehicle sustained minor injuries despite experiencing a similar energy level. In these cases, the crash severity was survivable without the guardrail penetration. The mean pre-impact speed, change in speed, and vehicular acceleration was $117 \mathrm{kph}, 20 \mathrm{kph}$, and $97 \mathrm{~m} / \mathrm{sec} 2$, respectively. The hybrid guardrail system deflected the vehicle without any penetration into the occupant compartment. The mean peak accelerations in crash tests were below injurious threshold levels. The present research shows that the hybrid guardrail system not only eliminated the intrusion into occupant survival space but also deflected the vehicle.
\end{abstract}

KEYWORDS:Transportation Safety, Biomechanics, Guardrail Accidents, Energy Absorbing Guardrail, Penetrating Guardrail Crash, Roadside Safety Hardware

\section{INTRODUCTION}

The highway safety efforts are geared to provide a safer roadway and roadside to mitigate injuries to occupants of motor vehicle crashes with exterior environments. One of the safety features along the roadside is the guardrail [1]. The function of the guardrail is to deflect the vehicle from environments such as trees, poles, side slopes and other hazards the vehicle may encounter in run-off road crashes. Guardrails are designed to dissipate crash impact energy typically seen in highway crashes. However, the guardrail poses a risk if it penetrates into the occupant compartment resulting in serious or fatal injuries. Annually, guardrail crashes involve approximately 1,000 fatalities and 35,000 injuries [2]. Griffith et al studied 161 cases of guardrail injuries involved in highway speed and found one of the contributory factors for serious or fatal injuries to be guardrail penetration [3]. While the penetrating structural intrusion from tractor-trailer crashes and associated protective measures have been addressed [4-5], the roadside guardrail penetration and injury mitigating efforts have not been researched in detail [6]. A better understanding of the mechanism of injuries and occupant protection in guardrail crashes will aid in the development of advanced roadside safety measures. The present work is designed to delineate the biomechanics of penetrating guardrail trauma by examining real-world crashes and to evaluate the biomechanical efficacy of the hybrid tension-compression guardrail system to protect occupants. 